\section{Utilização de serviços de saúde ambulatoriais no pós-parto por puérperas e recém-nascidos: dados do estudo Nascer no Brasil}

\author{
Use of outpatient health services by postpartum \\ women and newborns: data from the Birth in \\ Brazil study
}

\section{Utilización de servicios de salud ambulatorios en el posparto por parte de puérperas y recién nacidos: datos del estudio Nacer en Brasil}

Rosa Maria Soares Madeira Domingues 1

Barbara Almeida Soares Dias 2

Sonia Duarte de Azevedo Bittencourt 2

Marcos Augusto Bastos Dias 3

Jacqueline Alves Torres 4

Elenice Machado da Cunha 5

Maria do Carmo Leal 2

doi: 10.1590/0102-311X00119519

\section{Resumo}

Este trabalho tem por objetivo estimar a utilização de serviços de saúde ambulatoriais no pós-parto e verificar os fatores demográficos, socioeconômicos e obstétricos associados a este uso. Estudo nacional de base hospitalar, realizado em 2011-2012, com entrevistas de 23.894 mulheres. Foram calculadas as estimativas pontuais e os respectivos intervalos de confiança de oito indicadores de utilização de serviços de saúde com desempenho avaliado como "satisfatório" (75\%-100\%); "parcial" (50\%-74\%) e "insatisfatório" (< 50\%). Foi realizada regressão logística múltipla para verificar a associação entre as características das mulheres e cada um dos indicadores analisados. Quatro indicadores - "procura de serviço para consulta de revisão do parto" (73,9\%; IC95\%: 72,4-75,3); "procura de serviço para consulta do recém-nato" (91,6\%; IC95\%: 90,6-92,5); "vacinação com BCG” (99\%; IC95\%: 98, 7-99,2); e "vacinação contra hepatite B" (96,8\%; IC95\%: 96,0-97,5) foram considerados satisfatórios. A "coleta do teste de triagem neonatal na primeira semana de vida" foi considerada parcial (60,1\%; IC95\%: 57,6-62,6), e "consulta da mulher nos primeiros 15 dias após o parto" (37\%; IC95\%: 35,0-39,0), "consulta do recémnato nos primeiros sete dias de vida" (21,8\%; IC95\%: 20,2-23,5) e "recebimento do resultado da triagem neonatal no primeiro mês de vida" (29,8\%; IC95\%: 27,6-32,2) foram considerados insatisfatórios. Desigualdades regionais e sociais foram identificadas, com o pior desempenho de todos os indicadores nas regiões Norte e Nordeste e em mulheres mais vulneráveis, apontando para a necessidade de uma melhor organização e oferta dos serviços visando à redução de iniquidades.

Saúde Materno-Infantil; Serviços de Saúde Materno-Infantil; Cuidado da Criança; Período Pós-Parto

\section{Correspondência}

R. M. S. M. Domingues

Instituto Nacional de Infectologia Evandro Chagas, Fundação Oswaldo Cruz.

Av. Brasil 4365, Rio de Janeiro, RJ 21040-360, Brasil. rosa.domingues@ini.fiocruz.br

1 Instituto Nacional de Infectologia Evandro Chagas, Fundação Oswaldo Cruz, Rio de Janeiro, Brasil.

2 Escola Nacional de Saúde Pública Sergio Arouca, Fundação Oswaldo Cruz, Rio de Janeiro, Brasil.

3 Instituto Nacional de Saúde da Mulher, da Criança e do Adolescente Fernandes Figueira, Fundação Oswaldo Cruz, Rio de Janeiro, Brasil.

4 Agência Nacional de Saúde Suplementar, Rio de Janeiro, Brasil.

5 Escola Politécnica de Saúde Joaquim Venâncio, Fundação Oswaldo Cruz, Rio de Janeiro, Brasil. 


\section{Introdução}

O período pós-parto é especial e crítico para as mulheres. Elas experimentam, simultaneamente, mudanças hormonais e a expansão de seu papel e responsabilidades na família 1,2, com intensa dedicação requerida pelo recém-nascido para um crescimento e desenvolvimento ótimos.

O Ministério da Saúde brasileiro preconiza a "Primeira Semana de Saúde Integral" 3. Trata-se de uma estratégia da atenção básica em saúde, a ser realizada entre 7 e 10 dias após o parto, que tem por objetivos a avaliação do estado de saúde da mulher e do recém-nascido, a avaliação da interação da mãe com o bebê, a orientação para os cuidados básicos com o recém-nascido, a identificação de situações de risco ou intercorrências, a triagem neonatal, a triagem auditiva, a vacinação, a promoção e o apoio ao aleitamento materno, e a orientação sobre planejamento familiar 2.

No país, a assistência ao pré-natal e ao parto é praticamente universal, alcançando valores próximos a $100 \%$ de cobertura 4 . Entretanto, a realização da consulta de puerpério é ainda baixa. No único estudo de representatividade nacional, realizado em 2006, apenas 39,2\% das puérperas tiveram uma consulta realizada até os 42 dias após o parto 5 . Estudos locais, realizados nas regiões Sul e Sudeste do país, revelam proporções que variaram de 16,8\% a 77\% 6,7,8. Quanto à assistência ao recém-nascido, dados de abrangência nacional relativos à realização de consulta na primeira semana de vida não estão disponíveis. No Brasil, as coberturas da imunização infantil são elevadas (Departamento de Informática do SUS. Arquivos de DO reduzida para tabulação do Programa Nacional de Imunização. http:// tabnet.datasus.gov.br/cgi/deftohtm.exe?pni/cnv/cpniuf.def, acessado em 05/Dez/2018), porém permanecem como desafios a cobertura universal do "teste do pezinho" 9 e o comparecimento regular de crianças ao serviço de puericultura 10,11.

Diferentes fatores afetam a utilização de serviços de saúde, tais como a necessidade percebida de cuidados e a disponibilidade de serviços, sendo ambos afetados por fatores sociais, econômicos e demográficos 12. No país, dados da Pesquisa Nacional de Saúde (PNS), realizada em 2013, mostram maior utilização de serviços de saúde por indivíduos com idade superior a 60 anos, em domicílios com chefe de família com Ensino Superior, e residência nas regiões Sul e Sudeste 13. No âmbito específico do uso de serviços de saúde pós-natais por puérperas e seus recém-nascido, estudos nacionais e internacionais evidenciam menor utilização de serviços por mulheres residentes na Região Norte 5 , por residentes em áreas rurais 5,14, em mulheres com menor renda 7,14,15,16,17 ou de classe econômica mais baixa 5 , em mulheres 5,7,14,15,16,17,18 ou parceiros $14,17,18$ com menor escolaridade, por mulheres que se autodeclararam pretas ou pardas 7, adolescentes 5,7,18, multíparas 7,14,16, solteiras 7, fumantes 7, com morbidade na gestação 11,19, com pré-natal inadequado ou não realizado 7,14,17,20,21, e em usuárias de serviços públicos $5,7,15$.

Considerando-se a escassez de dados nacionais relativos à assistência no período pós-parto para a mulher e o recém-nascido, e a importância de estudos de base populacional para a obtenção de dados sobre acesso e uso de serviços visando ao planejamento e à gestão de políticas públicas, o presente estudo tem por objetivo estimar a utilização de serviços de saúde ambulatoriais no pós-parto por puérperas e recém-nascido assistidos durante o parto em maternidades públicas e privadas no Brasil, e verificar os fatores demográficos, socioeconômicos e obstétricos associados a este uso. A hipótese deste trabalho é a de que a utilização desses serviços é baixa e está associada a desigualdades regionais e socioeconômicas.

\section{Métodos}

O Nascer no Brasil é um estudo nacional de base hospitalar, realizado no período de fevereiro de 2011 a outubro de 2012, que avaliou a assistência pré-natal, ao parto e ao pós-parto de mulheres com parto hospitalar que tiveram como desfecho da gestação um recém-nato vivo com qualquer peso ou idade gestacional, ou um feto morto com peso > 500g ou idade gestacional maior que 22 semanas.

A amostra foi selecionada em três etapas. Na primeira, hospitais com mais de 500 partos por ano foram estratificados de acordo com as cinco macrorregiões do país, localização (capital ou interior) e tipo de serviço (público, misto ou privado), sendo selecionados 266 hospitais com uma probabilidade de seleção proporcional ao número de partos em cada um dos estratos em 2007. Na segunda etapa, 
foi utilizado um método de amostragem inversa para calcular o número de dias necessários para entrevistar 90 puérperas em cada hospital, considerando-se um mínimo de sete dias de permanência da equipe de pesquisa em cada serviço. A terceira consistiu na seleção de puérperas. Em hospitais com menos de 90 partos por semana, todas as mulheres que preencheram os critérios de inclusão foram selecionadas sequencialmente até que 90 entrevistas fossem alcançadas. Em hospitais com mais de 90 partos por semana, foi realizada uma amostra aleatória de todas as mulheres elegíveis hospitalizadas durante os sete dias em que a equipe de pesquisa se encontrava no hospital. No total, foram entrevistadas 23.894 mulheres. Mais informações sobre o processo amostral do estudo Nascer no Brasil podem ser obtidos em Vasconcellos et al. 22.

A coleta de dados incluiu entrevista face a face com as puérperas realizada durante a internação hospitalar, no mínimo seis horas após o parto; extração de dados dos prontuários maternos e do recém-nascido após a alta hospitalar; extração de dados do cartão de pré-natal, quando disponível; e duas entrevistas telefônicas realizadas após a alta hospitalar. Toda a coleta de dados foi realizada por profissionais treinados pela equipe de coordenação central da pesquisa Nascer no Brasil, utilizando instrumentos desenvolvidos especificamente para este trabalho. Mais informações sobre o desenho do estudo Nascer no Brasil e os procedimentos usados na coleta de dados podem ser obtidos em Leal et al. 23 .

Para o presente estudo, utilizamos dados obtidos na entrevista hospitalar e na primeira entrevista telefônica. Essa entrevista telefônica foi realizada no mínimo 43 dias após o parto, com mediana de 81 dias (intervalo interquartil 55 a 117 dias). A entrevista foi realizada por profissionais treinados e durava aproximadamente 10 minutos. Foi usado um questionário fechado contendo perguntas sobre aleitamento materno, morbimortalidade materna e neonatal, e utilização de serviços de saúde da atenção básica e hospitalar após a alta. Foram entrevistadas 16.109 mulheres, com uma taxa de resposta de $67,4 \%$ em relação às puérperas entrevistadas no pós-parto imediato.

Neste artigo, foi verificada a utilização dos serviços de saúde da atenção básica por mulheres e recém-natos após a alta hospitalar, por meio dos seguintes indicadores baseados nas recomendações do Ministério da Saúde:

a) Procura de serviço de saúde para a consulta de revisão do parto pela puérpera;

b) Realização de consulta de revisão do parto nos primeiros 15 dias pós-parto;

c) Procura de serviço de saúde pela mulher para a realização da consulta de rotina de acompanhamento do recém-nascido;

d) Realização da primeira consulta de rotina de seguimento do recém-nascido na primeira semana de vida;

e) Vacinação do recém-nascido com a vacina BCG;

f) Vacinação do recém-nascido com a primeira dose da vacina contra hepatite B;

g) Realização do teste do pezinho nos primeiros sete dias de vida do recém-nascido;

h) Recebimento dos resultados do teste do pezinho no primeiro mês de vida do recém-nascido.

Foram consideradas elegíveis para esta análise mulheres com gestações únicas e com recém-nascido vivo na alta hospitalar. Para a avaliação do indicador "realização de consulta de revisão do parto nos primeiros 15 dias pós-parto" foram excluídas as mulheres com internação hospitalar igual ou superior a 15 dias, e para o indicador "realização da primeira consulta de rotina do recém-nascido na primeira semana de vida" foram excluídos os recém-natos que permaneceram internados por sete ou mais dias.

$\mathrm{Na}$ análise descritiva serão apresentadas as características das mulheres incluídas nesta análise e o cálculo das estimativas pontuais com o respectivo intervalo de $95 \%$ de confiança (IC95\%) dos oito indicadores de utilização dos serviços pela puérpera e recém-nascido. A adequação do uso dos serviços foi avaliada pela proporção de mulheres que respondeu "sim" em relação ao total de elegíveis, com o seguinte padrão de avaliação: "satisfatório" = 75\%-100\%; "parcial" = 50\%-74\%; e "insatisfatório" = abaixo de $50 \% 24$.

Para verificar os fatores associados à utilização dos serviços de atenção à mulher e ao recémnato após o parto, foram realizadas regressões logísticas simples e múltipla para cada um dos oito indicadores utilizados. Foram analisadas as variáveis região de residência (Norte, Nordeste, Sudeste, Sul, Centro-oeste), idade materna ( $<20$ anos, 20-34, $\geq 35$ anos), cor da pele autorreferida (branca, preta, parda, amarela, indígena), escolaridade materna (Ensino Fundamental incompleto, Ensino 
Fundamental completo, Ensino Médio, Ensino Superior e mais), paridade (primípara ou não), classe econômica segundo a classificação da ABIPEME 25 (A/B, C, D/E, sendo as classes A/B as de melhor condição econômica), plano de saúde (sim/não), adequação da assistência pré-natal (sim ou não, sendo considerado adequado o início do pré-natal no primeiro trimestre gestacional e o número adequado de consultas para a idade gestacional no parto, tendo como padrão um mínimo de seis consultas para uma gestação a termo); e ter recebido orientação na maternidade para o comparecimento a um serviço de saúde para realizar consulta de revisão 7 a 10 dias após o parto.

Para lidar com as perdas de seguimentos na entrevista telefônica, um modelo de regressão logística foi ajustado para estimar a probabilidade de cada mulher participante no início do estudo responder à entrevista telefônica, utilizando um conjunto de variáveis que diferenciavam os grupos de respondentes e não respondentes. Com base nesse modelo, foram calculados pesos amostrais específicos para a análise dos dados da entrevista telefônica, com o intuito de compensar a tendência de mulheres com certas características (como ser mais jovem e de menor escolaridade) de ter taxas mais baixas de resposta, afetando a probabilidade de resposta em um estrato específico. A justificativa para a aplicação de pesos de não resposta é a suposição de que os não respondentes teriam fornecido respostas semelhantes, em média, às respostas dos entrevistados para cada estrato e categoria de ajuste. Maiores detalhes sobre o procedimento de ponderação e calibração usados podem ser obtidos em Vasconcellos et al. 22. Toda a análise estatística foi realizada usando-se o software SPSS versão 17 (https://www. ibm.com/), com a utilização da ponderação dos dados e incorporação do efeito de desenho, considerando-se o processo complexo da amostragem.

Este estudo foi aprovado pelo Comitê de Ética em Pesquisa da Escola Nacional de Saúde Pública Sergio Arouca, Fundação Oswaldo Cruz (ENSP/Fiocruz), parecer no 92/2010. Todos os cuidados foram adotados visando a garantir o sigilo e confidencialidade das informações. Antes da realização de cada entrevista, foi obtido o consentimento após a leitura do Termo de Consentimento Livre e Esclarecido.

\section{Resultados}

Foram consideradas elegíveis para esta análise 23.368 mulheres (97,8\% do total de entrevistadas). A maior parte delas residia nas regiões Sudeste $(42,7 \%)$ e Nordeste $(28,7 \%)$ do país. A média de idade foi de 25,7 anos, sendo 18 ,7\% adolescentes (<20 anos) e 10,3\% com $\geq 35$ anos. A maioria das entrevistadas $(56,8 \%)$ declarou ter cor da pele parda, e uma pequena parcela declarou cor da pele amarela $(1,1 \%)$ e indígena (0,3\%). Quase a metade (49,5\%) apresentava Ensino Fundamental e 9\% possuíam o Ensino Superior. Um pouco mais de $80 \%$ das mulheres viviam com o companheiro e 48,4\% eram primíparas. Em relação à classificação econômica, 54,2\% pertenciam à classe $\mathrm{C}$ e 72,8\% não tinham plano de saúde. Aproximadamente dois terços do total de entrevistadas apresentaram um pré-natal adequado, considerando época de início e número de consultas, e 69,9\% referiram ter recebido orientação na maternidade para comparecer a um serviço de saúde após o parto (Tabela 1).

As Tabelas 2, 3, 4 e 5 apresentam as estimativas de utilização dos serviços de saúde no pós-parto e os fatores associados a este uso. A procura por um serviço de saúde para a consulta de revisão do parto foi referida por 73,9\% das mulheres (IC95\%: 72,4-75,3) e 37\% (IC95\%: 35,0-39,0) relataram ter conseguido o atendimento nos primeiros 15 dias após o parto. A maior procura foi relatada nas regiões Sul, Sudeste e Centro-oeste, por mulheres com nível médio ou superior de escolaridade, que possuíam plano de saúde, que tiveram uma assistência pré-natal adequada e que receberam orientação na maternidade para procurar um serviço de saúde após o parto (Tabela 2). Padrão semelhante foi observado para a obtenção do atendimento nos primeiros 15 dias após o parto, exceto para a Região Centro-oeste, que foi semelhante às regiões Norte e Nordeste. Foi verificado também menor recebimento de consultas por mulheres pardas e aquelas de classe econômica mais baixa (classes C, D e E). Mulheres indígenas apresentaram a menor proporção de recebimento de consulta no pós-parto, sendo esta diferença não significativa provavelmente em decorrência do pequeno número de mulheres nesta categoria. Para ambos os indicadores, a variável com maior força de associação foi ter recebido orientação na maternidade para comparecer a um serviço de saúde após o parto (OR = 7,76; IC95\%: 6,85-8,80 e OR = 4,40; IC95\%: 3,85-5,04, respectivamente) (Tabela 2). 


\section{Tabela 1}

Distribuição das características socioeconômicas, demográficas e obstétricas das mulheres entrevistadas. Brasil, 2011-2012.

\begin{tabular}{|c|c|c|}
\hline Características & $\begin{array}{c}n \\
(N=23.368)\end{array}$ & $\%$ \\
\hline \multicolumn{3}{|l|}{ Região } \\
\hline Norte & 2.227 & 9,5 \\
\hline Nordeste & 6.709 & 28,7 \\
\hline Sudeste & 9.988 & 42,7 \\
\hline Sul & 2.921 & 12,5 \\
\hline Centro-oeste & 1.522 & 6,5 \\
\hline \multicolumn{3}{|l|}{ Idade (anos) } \\
\hline 35 ou mais & 2.411 & 10,3 \\
\hline $20-34$ & 16.593 & 71,0 \\
\hline $12-19$ & 4.357 & 18,7 \\
\hline \multicolumn{3}{|l|}{ Cor da pele } \\
\hline Branca & 7.830 & 33,5 \\
\hline Parda & 13.261 & 56,8 \\
\hline Preta & 1.935 & 8,3 \\
\hline Amarela & 260 & 1,1 \\
\hline Indígena & 79 & 0,3 \\
\hline \multicolumn{3}{|l|}{ Escolaridade } \\
\hline Ensino Fundamental incompleto & 5.337 & 22,9 \\
\hline Ensino Fundamental completo & 6.187 & 26,6 \\
\hline Ensino Médio completo & 9.655 & 41,5 \\
\hline Ensino Superior completo e mais & 2.086 & 9,0 \\
\hline \multicolumn{3}{|l|}{ Situação conjugal } \\
\hline Com companheiro & 19.071 & 81,7 \\
\hline Sem companheiro & 4.265 & 18,3 \\
\hline \multicolumn{3}{|l|}{ Primípara } \\
\hline Sim & 11.317 & 48,4 \\
\hline Não & 12.050 & 51,6 \\
\hline \multicolumn{3}{|l|}{ Classe econômica } \\
\hline$D / E$ & 4.977 & 21,5 \\
\hline C & 12.560 & 54,2 \\
\hline$A / B$ & 5.635 & 24,3 \\
\hline \multicolumn{3}{|l|}{ Plano de saúde } \\
\hline Não & 16.986 & 72,8 \\
\hline Sim & 6.355 & 27,2 \\
\hline \multicolumn{3}{|l|}{ Adequação do pré-natal } \\
\hline Inadequado & 7.130 & 31,2 \\
\hline Adequado & 15.691 & 68,8 \\
\hline \multicolumn{3}{|c|}{$\begin{array}{l}\text { Orientada pela maternidade a comparecer a } \\
\text { serviço de pós-parto }\end{array}$} \\
\hline Não & 6.999 & 30,1 \\
\hline Sim & 16.269 & 69,9 \\
\hline
\end{tabular}

Nota: pequenas diferenças nos totais de cada variável devido ao número de informaç̧̃̃es faltantes: adequação pré-natal: missing $=2,3 \%$; demais variáveis: missing $<1,0 \%$. 
Tabela 2

Fatores associados à procura e realização de consulta de pós-parto. Brasil, 2011-2012.

\begin{tabular}{|c|c|c|c|c|c|c|c|c|c|c|}
\hline \multirow[t]{2}{*}{ Característica da mulher } & \multicolumn{5}{|c|}{$\begin{array}{l}\text { Procurou serviço de saúde para a } \\
\text { consulta de revisão do parto } \\
\qquad(\mathrm{N}=\mathbf{2 3 . 3 6 8})\end{array}$} & \multicolumn{5}{|c|}{$\begin{array}{l}\text { Realização de consulta de revisão do parto } \\
\text { nos primeiros } 15 \text { dias pós-parto } \\
(N=23.190) \text { * }\end{array}$} \\
\hline & $\%$ & $\mathbf{R C b}$ & IC95\% & $\mathrm{RCa}$ & IC95\% & $\%$ & $\mathrm{RCb}$ & IC95\% & $\mathrm{RCa}$ & IC95\% \\
\hline Total & 73,9 & & & & & 37,0 & & & & \\
\hline \multicolumn{11}{|l|}{ Região } \\
\hline Norte & 57,2 & 1,00 & ** & 1,00 & ** & 25,6 & 1,00 & ** & 1,00 & ** \\
\hline Nordeste & 62,8 & 1,26 & $0,99-1,60$ & 1,18 & $0,91-1,53$ & 28,6 & 1,16 & $0,95-1,43$ & 0,99 & $0,78-1,25$ \\
\hline Sudeste & 80,9 & 3,18 & $2,53-4,00$ & 2,12 & $1,64-2,73$ & 40,8 & 2,00 & $1,62-2,48$ & 1,27 & $1,01-1,58$ \\
\hline Sul & 87,0 & 5,01 & $3,78-6,63$ & 2,94 & $2,20-3,92$ & 50,4 & 2,96 & $2,42-3,62$ & 1,65 & $1,31-2,08$ \\
\hline Centro-oeste & 75,6 & 2,32 & $1,80-2,99$ & 1,66 & $1,22-2,26$ & 38,9 & 1,86 & $1,44-2,40$ & 1,30 & $0,94-1,79$ \\
\hline \multicolumn{11}{|l|}{ Idade (anos) } \\
\hline 35 ou mais & 77,6 & 1,00 & ** & & & 42,8 & 1,00 & ** & & \\
\hline $20-34$ & 75,2 & 0,57 & $0,45-0,72$ & & & 38,2 & 0,54 & $0,43-0,69$ & & \\
\hline $12-19$ & 66,5 & 0,87 & $0,72-1,06$ & & & 29,0 & 0,83 & $0,70-0,97$ & & \\
\hline \multicolumn{11}{|l|}{ Cor da pele autorreferida } \\
\hline Branca & 79,7 & 1,00 & ** & & & 45,5 & 1,00 & ** & 1,00 & $* \star *$ \\
\hline Parda & 70,7 & 0,61 & $0,54-0,70$ & & & 32,8 & 0,58 & $0,52-0,66$ & 0,85 & $0,75-0,95$ \\
\hline Preta & 72,2 & 0,66 & $0,55-0,80$ & & & 32,3 & 0,57 & $0,47-0,70$ & 0,88 & $0,72-1,07$ \\
\hline Amarela & 75,4 & 0,78 & $0,54-1,13$ & & & 32,6 & 0,58 & $0,36-0,94$ & 0,69 & $0,40-1,21$ \\
\hline Indígena & 69,3 & 0,58 & $0,31-1,09$ & & & 22,3 & 0,34 & $0,15-0,78$ & 0,55 & $0,21-1,47$ \\
\hline \multicolumn{11}{|l|}{ Escolaridade } \\
\hline $\begin{array}{l}\text { Ensino Fundamental } \\
\text { incompleto }\end{array}$ & 67,0 & 1,00 & ** & 1,00 & ** & 28,1 & 1,00 & ** & 1,00 & ** \\
\hline $\begin{array}{l}\text { Ensino Fundamental } \\
\text { completo }\end{array}$ & 69,5 & 1,13 & $0,95-1,33$ & 1,10 & $0,93-1,30$ & 31,0 & 1,15 & $0,96-1,37$ & 1,04 & $0,88-1,23$ \\
\hline Ensino Médio completo & 77,6 & 1,71 & $1,48-1,97$ & 1,45 & $1,24-1,70$ & 40,1 & 1,71 & $1,44-2,04$ & 1,25 & $1,05-1,49$ \\
\hline $\begin{array}{l}\text { Ensino Superior completo } \\
\text { e mais }\end{array}$ & 87,2 & 3,36 & $2,63-4,29$ & 2,15 & $1,64-2,82$ & 62,4 & 4,23 & $3,34-5,36$ & 2,16 & $1,69-2,76$ \\
\hline \multicolumn{11}{|l|}{ Primípara } \\
\hline Não & 73,8 & 1,00 & $\#$ & & & 36,4 & 1,00 & $\#$ & & \\
\hline Sim & 74,0 & 1,01 & $0,92-1,11$ & & & 37,5 & 1,05 & $0,96-1,15$ & & \\
\hline \multicolumn{11}{|l|}{ Classe econômica } \\
\hline$D / E$ & 64,1 & 1,00 & ** & & & 25,7 & 1,00 & ** & 1,00 & ** \\
\hline C & 73,0 & 1,52 & $1,33-1,72$ & & & 34,3 & 1,51 & $1,34-1,70$ & 1,13 & $0,99-1,29$ \\
\hline $\mathrm{A} / \mathrm{B}$ & 84,5 & 3,05 & $2,57-3,62$ & & & 52,5 & 3,20 & $2,74-3,75$ & 1,42 & $1,20-1,68$ \\
\hline \multicolumn{11}{|l|}{ Plano de saúde } \\
\hline Não & 69,8 & 1,00 & ** & 1,00 & $\# \#$ & 31,2 & 1,00 & ** & 1,00 & \\
\hline Sim & 84,5 & 2,35 & $2,02-2,73$ & 1,32 & $1,12-1,54$ & 52,4 & 2,43 & $2,08-2,85$ & 1,42 & $1,22-1,66$ \\
\hline \multicolumn{11}{|l|}{ Adequação do pré-natal } \\
\hline Inadequado & 67,1 & 1,00 & ** & 1,00 & ** & 29,8 & 1,00 & ** & 1,00 & ** \\
\hline Adequado & 77,0 & 1,64 & $1,46-1,83$ & 1,33 & $1,17-1,50$ & 40,2 & 1,59 & $1,41-1,78$ & 1,22 & $1,09-1,36$ \\
\hline \multicolumn{11}{|l|}{$\begin{array}{l}\text { Orientada a comparecer a } \\
\text { serviço de pós-parto }\end{array}$} \\
\hline Não & 42,8 & 1,00 & $* *$ & 1,00 & ** & 14,6 & 1,00 & ** & 1,00 & ** \\
\hline Sim & 87,2 & 9,08 & $7,99-10,33$ & 7,76 & $6,85-8,80$ & 46,7 & 5,12 & $4,48-5,84$ & 4,40 & $3,85-5,04$ \\
\hline
\end{tabular}

IC95\%: intervalo de 95\% de confiança; RCa: razão de chances ajustada; RCb: razão de chances bruta.

* Apenas entre aquelas com alta hospitalar menos de 15 dias após o parto;

** $\mathrm{p}<0,001$;

$* * * \mathrm{p}<0,05$;

$\# p>0,20$;

$\# p=0,001$. 
Tabela 3

Fatores associados à procura e realização de consulta do recém-nato. Brasil, 2011-2012.

\begin{tabular}{|c|c|c|c|c|c|c|c|c|c|c|}
\hline \multirow[t]{2}{*}{$\begin{array}{l}\text { Característica } \\
\text { da mulher }\end{array}$} & \multicolumn{5}{|c|}{$\begin{array}{l}\text { Procura de serviço de saúde para realização da } \\
\text { consulta de rotina de acompanhamento do recém-nascido } \\
\qquad(\mathrm{N}=\mathbf{2 3 . 3 6 8 )}\end{array}$} & \multicolumn{5}{|c|}{$\begin{array}{l}\text { Realização da primeira consulta de rotina de seguimento } \\
\text { do recém-nascido na primeira semana de vida } \\
\qquad(\mathrm{N}=\mathbf{2 2 . 0 2 3}) \text { * }\end{array}$} \\
\hline & $\%$ & $\mathrm{RCb}$ & IC95\% & $\mathrm{RCa}$ & IC95\% & $\%$ & $\mathbf{R C b}$ & IC95\% & $\mathrm{RCa}$ & IC95\% \\
\hline Total & 91,6 & & & & & 21,8 & & & & \\
\hline \multicolumn{11}{|l|}{ Região } \\
\hline Norte & 83,1 & 1,00 & ** & 1,00 & ** & 13,0 & 1,00 & ** & 1,00 & ** \\
\hline Nordeste & 86,5 & 1,30 & $0,93-1,82$ & 1,11 & $0,80-1,56$ & 14,8 & 1,17 & $0,83-1,64$ & 1,06 & $0,75-1,50$ \\
\hline Sudeste & 94,7 & 3,60 & $2,56-5,07$ & 2,44 & $1,74-3,44$ & 25,0 & 2,24 & $1,69-2,98$ & 1,70 & $1,25-2,30$ \\
\hline Sul & 96,7 & 6,02 & $4,13-8,79$ & 3,48 & $2,34-5,16$ & 33,0 & 3,30 & $2,50-4,35$ & 2,17 & $1,60-2,95$ \\
\hline Centro-oeste & 95,7 & 4,56 & $2,91-7,14$ & 3,26 & $1,99-5,32$ & 22,7 & 1,97 & $1,44-2,71$ & 1,56 & $1,11-2,19$ \\
\hline \multicolumn{11}{|l|}{ Idade (anos) } \\
\hline 35 ou mais & 93,7 & 1,00 & $* *$ & 1,00 & $* * *$ & 24,5 & 1,00 & ** & & \\
\hline $20-34$ & 92,3 & 0,81 & $0,60-1,10$ & 0,94 & $0,67-1,31$ & 22,7 & 0,91 & $0,79-1,04$ & & \\
\hline $12-19$ & 87,4 & 0,47 & $0,34-0,66$ & 0,61 & $0,41-0,90$ & 16,9 & 0,63 & $0,53-0,74$ & & \\
\hline \multicolumn{11}{|l|}{$\begin{array}{l}\text { Cor da pele } \\
\text { autorreferida }\end{array}$} \\
\hline Branca & 94,2 & 1,00 & $* *$ & & & 29,5 & 1,00 & ** & 1,00 & ** \\
\hline Parda & 90,0 & 0,55 & $0,44-0,68$ & & & 18,0 & 0,52 & $0,46-0,60$ & 0,78 & $0,68-0,89$ \\
\hline Preta & 91,7 & 0,68 & $0,49-0,93$ & & & 17,3 & 0,50 & $0,38-0,65$ & 0,77 & $0,58-1,01$ \\
\hline Amarela & 88,3 & 0,46 & $0,27-0,79$ & & & 20,5 & 0,62 & $0,40-0,96$ & 0,71 & $0,46-1,09$ \\
\hline Indígena & 93,2 & 0,84 & $0,30-2,29$ & & & 2,7 & 0,07 & $0,02-0,27$ & 0,10 & $0,02-0,40$ \\
\hline \multicolumn{11}{|l|}{ Escolaridade } \\
\hline \multicolumn{11}{|l|}{ Ensino } \\
\hline $\begin{array}{l}\text { Fundamental } \\
\text { incompleto }\end{array}$ & 87,0 & 1,00 & ** & 1,00 & $\#$ & 15,5 & 1,00 & ** & 1,00 & ** \\
\hline \multicolumn{11}{|l|}{ Ensino } \\
\hline $\begin{array}{l}\text { Fundamental } \\
\text { completo }\end{array}$ & 89,1 & 1,22 & $1,03-1,46$ & 1,00 & $0,81-1,22$ & 18,8 & 1,26 & $1,03-1,55$ & 1,06 & $0,87-1,31$ \\
\hline $\begin{array}{l}\text { Ensino Médio } \\
\text { completo }\end{array}$ & 94,3 & 2,47 & $2,12-2,88$ & 1,33 & $1,08-1,63$ & 22,9 & 1,62 & $1,37-1,91$ & 1,12 & $0,94-1,34$ \\
\hline \multicolumn{11}{|l|}{ Ensino } \\
\hline $\begin{array}{l}\text { Superior } \\
\text { completo e } \\
\text { mais }\end{array}$ & 97,6 & 6,07 & $3,90-9,46$ & 1,48 & $0,89-2,47$ & 40,2 & 3,66 & $2,91-4,59$ & 1,82 & $1,47-2,25$ \\
\hline \multicolumn{11}{|l|}{ Primípara } \\
\hline Não & 90,0 & 1,00 & ** & 1,00 & $\star \star$ & 20,3 & 1,00 & ** & 1,00 & $\# \#$ \\
\hline Sim & 93,2 & 1,53 & $1,32-1,76$ & 1,74 & $1,44-2,10$ & 23,4 & 1,20 & $1,07-1,33$ & 1,15 & $1,04-1,26$ \\
\hline \multicolumn{11}{|l|}{$\begin{array}{l}\text { Classe } \\
\text { econômica }\end{array}$} \\
\hline$D / E$ & 85,3 & 1,00 & ** & 1,00 & ** & 13,2 & 1,00 & ** & 1,00 & ** \\
\hline C & 91,6 & 1,87 & $1,58-2,22$ & 1,31 & $1,08-1,60$ & 19,8 & 1,62 & $1,35-1,94$ & 1,25 & $1,03-1,51$ \\
\hline $\mathrm{A} / \mathrm{B}$ & 96,9 & 5,37 & $3,83-7,54$ & 2,30 & $1,70-3,13$ & 33,4 & 3,29 & $2,69-4,02$ & 1,60 & $1,30-1,98$ \\
\hline \multicolumn{11}{|l|}{ Plano de saúde } \\
\hline Não & 89,4 & 1,00 & $* *$ & 1,00 & $* *$ & 17,8 & 1,00 & $* *$ & 1,00 & $* *$ \\
\hline Sim & 97,3 & 4,30 & $3,29-5,61$ & 1,81 & $1,37-2,40$ & 32,4 & 2,22 & $1,88-2,61$ & 1,34 & $1,15-1,56$ \\
\hline
\end{tabular}

(continuação) 
Tabela 3 (continua)

\begin{tabular}{|c|c|c|c|c|c|c|c|c|c|c|}
\hline \multirow[t]{2}{*}{$\begin{array}{l}\text { Característica } \\
\text { da mulher }\end{array}$} & \multicolumn{5}{|c|}{$\begin{array}{l}\text { Procura de serviço de saúde para realização da } \\
\text { consulta de rotina de acompanhamento do recém-nascido } \\
\qquad(\mathrm{N}=23.368)\end{array}$} & \multicolumn{5}{|c|}{$\begin{array}{l}\text { Realização da primeira consulta de rotina de seguimento } \\
\text { do recém-nascido na primeira semana de vida } \\
\qquad(\mathrm{N}=22.023) \text { * }\end{array}$} \\
\hline & $\%$ & $\mathrm{RCb}$ & IC95\% & $\mathrm{RCa}$ & IC95\% & $\%$ & $\mathrm{RCb}$ & IC95\% & $\mathrm{RCa}$ & IC95\% \\
\hline \multicolumn{11}{|l|}{$\begin{array}{l}\text { Adequação do } \\
\text { pré-natal }\end{array}$} \\
\hline Inadequado & 88,2 & 1,00 & $\star \star$ & 1,00 & $\star \star$ & 18,7 & 1,00 & $\star \star$ & & \\
\hline Adequado & 93,2 & 1,82 & $1,56-2,12$ & 1,24 & $1,06-1,46$ & 23,2 & 1,31 & $1,17-1,48$ & & \\
\hline \multicolumn{11}{|l|}{$\begin{array}{l}\text { Orientada a } \\
\text { comparecer a } \\
\text { serviço de } \\
\text { pós-parto }\end{array}$} \\
\hline Não & 85,4 & 1,00 & $\star \star$ & 1,00 & $\star \star$ & 14,7 & 1,00 & $\star \star$ & 1,00 & $\star \star$ \\
\hline Sim & 94,3 & 2,83 & $2,42-3,31$ & 2,03 & $1,72-2,40$ & 24,9 & 1,93 & $1,69-2,20$ & 1,53 & $1,33-1,75$ \\
\hline
\end{tabular}

IC95\%: intervalo de 95\% de confiança; RCa: razão de chances ajustada; RCb: razão de chances bruta.

* Apenas para recém-natos com tempo de internação inferior a 7 dias.

** $\mathrm{p}<0,001$

$\star * * \mathrm{p}=0,002$

$\# \mathrm{p}=0,009$;

$\#$ \# $=0,005$.

\section{Tabela 4}

Fatores associados à vacinação com BCG e hepatite B após o nascimento. Brasil, 2011-2012.

\begin{tabular}{|c|c|c|c|c|c|c|c|c|c|c|}
\hline \multirow[t]{2}{*}{ Característica da mulher } & \multicolumn{5}{|c|}{$\begin{array}{l}\text { Vacinação do recém-nascido com } \\
\qquad \begin{array}{c}\text { a vacina BCG } \\
(N=23.368)\end{array}\end{array}$} & \multicolumn{5}{|c|}{$\begin{array}{l}\text { Vacinação do recém-nascido com a primeira } \\
\text { dose da vacina contra hepatite B } \\
\qquad(N=23.368)\end{array}$} \\
\hline & $\%$ & $\mathbf{R C b}$ & IC95\% & $\mathrm{RCa}$ & IC95\% & $\%$ & $\mathbf{R C b}$ & IC95\% & $\mathrm{RCa}$ & IC95\% \\
\hline Total & 99,0 & & & & & 96,8 & & & & \\
\hline \multicolumn{11}{|l|}{ Região } \\
\hline Norte & 98,7 & 1,00 & * & & & 98,0 & 1,00 & $\star \star$ & 1,00 & $\star \star$ \\
\hline Nordeste & 99,1 & 1,46 & $0,72-2,98$ & & & 98,3 & 1,22 & $0,60-2,51$ & 1,24 & $0,61-2,51$ \\
\hline Sudeste & 98,9 & 1,15 & $0,58-2,28$ & & & 95,1 & 0,41 & $0,22-0,77$ & 0,39 & $0,21-0,72$ \\
\hline Sul & 99,4 & 2,05 & $0,96-4,39$ & & & 97,2 & 0,72 & $0,40-1,28$ & 0,71 & $0,40-1,25$ \\
\hline Centro-oeste & 99,4 & 2,03 & $0,80-5,16$ & & & 98,8 & 1,67 & $0,71-3,90$ & 1,61 & $0,69-3,77$ \\
\hline \multicolumn{11}{|l|}{ Idade (anos) } \\
\hline 35 ou mais & 99,4 & 1,00 & $\star \star \star$ & & & 97,0 & 1,00 & * & & \\
\hline $20-34$ & 99,1 & 0,70 & $0,31-1,59$ & & & 96,8 & 0,91 & $0,63-1,31$ & & \\
\hline $12-19$ & 98,4 & 0,39 & $0,17-0,88$ & & & 96,8 & 0,91 & $0,57-1,48$ & & \\
\hline \multicolumn{11}{|l|}{ Cor da pele autorreferida } \\
\hline Branca & 99,3 & 1,00 & * & & & 96,6 & 1,00 & * & & \\
\hline Parda & 98,8 & 0,58 & $0,34-0,98$ & & & 97,0 & 1,14 & $0,77-1,70$ & & \\
\hline Preta & 99,1 & 0,76 & $0,30-1,89$ & & & 96,4 & 0,96 & $0,39-2,35$ & & \\
\hline Amarela & 98,6 & 0,48 & $0,08-2,95$ & & & 97,3 & 1,27 & $0,42-3,87$ & & \\
\hline Indígena & 98,0 & 0,33 & $0,04-2,60$ & & & 95,0 & 0,68 & $0,15-3,11$ & & \\
\hline
\end{tabular}

(continuação) 


\begin{tabular}{|c|c|c|c|c|c|c|c|c|c|c|}
\hline \multirow[t]{2}{*}{ Característica da mulher } & \multicolumn{5}{|c|}{$\begin{array}{l}\text { Vacinação do recém-nascido com } \\
\text { a vacina BCG } \\
(\mathbf{N}=23.368)\end{array}$} & \multicolumn{5}{|c|}{$\begin{array}{l}\text { Vacinação do recém-nascido com a primeira } \\
\text { dose da vacina contra hepatite B } \\
\qquad(N=23.368)\end{array}$} \\
\hline & $\%$ & $\mathrm{RCb}$ & $\mathrm{IC95 \%}$ & $\mathrm{RCa}$ & IC95\% & $\%$ & $\mathbf{R C b}$ & IC95\% & $\mathrm{RCa}$ & IC95\% \\
\hline \multicolumn{11}{|l|}{ Escolaridade } \\
\hline Ensino Fundamental incompleto & 98,4 & 1,00 & $* \star \star$ & 1,00 & $* * *$ & 95,7 & 1,00 & $* \star *$ & 1,00 & $* \star \star$ \\
\hline Ensino Fundamental completo & 99,2 & 2,02 & $1,23-3,32$ & 2,14 & $1,32-3,47$ & 96,7 & 1,32 & $0,94-1,86$ & 1,42 & $1,01-1,99$ \\
\hline Ensino Médio completo & 99,1 & 1,88 & $0,96-3,68$ & 1,82 & $0,92-3,60$ & 97,3 & 1,64 & $1,16-2,32$ & 1,85 & $1,33-2,57$ \\
\hline Ensino Superior completo e mais & 99,5 & 2,93 & $1,08-7,92$ & 1,91 & $0,57-6,35$ & 97,2 & 1,57 & $0,87-2,84$ & 1,69 & $0,96-2,99$ \\
\hline \multicolumn{11}{|l|}{ Primípara } \\
\hline Não & 99,3 & 1,00 & $\star \star *$ & 1,00 & $* * *$ & 96,5 & 1,00 & $\#$ & & \\
\hline Sim & 98,7 & 0,57 & $0,34-0,95$ & 0,48 & $0,30-0,77$ & 97,1 & 1,23 & $0,96-1,58$ & & \\
\hline \multicolumn{11}{|l|}{ Classe econômica } \\
\hline $\mathrm{D} / \mathrm{E}$ & 98,6 & 1,00 & ** & & & 96,8 & 1,00 & * & & \\
\hline c & 98,9 & 1,28 & $0,75-2,19$ & & & 96,7 & 0,95 & $0,61-1,49$ & & \\
\hline $\mathrm{A} / \mathrm{B}$ & 99,6 & 3,46 & $1,86-6,45$ & & & 97,0 & 1,08 & $0,66-1,75$ & & \\
\hline \multicolumn{11}{|l|}{ Plano de saúde } \\
\hline Não & 98,8 & 1,00 & ** & 1,00 & $* * *$ & 96,5 & 1,00 & $\# \#$ & & \\
\hline Sim & 99,6 & 2,82 & $1,59-4,98$ & 2,60 & $1,21-5,61$ & 97,6 & 1,47 & $1,00-2,18$ & & \\
\hline \multicolumn{11}{|l|}{ Adequação do pré-natal } \\
\hline Inadequado & 98,9 & 1,00 & * & & & 96,4 & 1,00 & * & & \\
\hline Adequado & 99,1 & 1,26 & $0,74-2,15$ & & & 97,0 & 1,18 & $0,85-1,63$ & & \\
\hline \multicolumn{11}{|l|}{$\begin{array}{l}\text { Orientada a comparecer a serviço } \\
\text { de pós-parto }\end{array}$} \\
\hline Não & 98,9 & 1,00 & * & & & 97,0 & 1,00 & * & & \\
\hline Sim & 99,1 & 1,24 & $0,75-2,06$ & & & 96,9 & 0,94 & $0,74-1,21$ & & \\
\hline
\end{tabular}

IC95\%: intervalo de 95\% de confiança; RCa: razão de chances ajustada; RCb: razão de chances bruta.

$* p>0,20$;

** $p<0,001$;

$* * * p<0,05$

$\# p=0,103$;

$\#$ \# $=0,052$.

Em relação aos serviços de atenção à criança, 91,6\% (IC95\%: 90,6-92,5) das mulheres referiram ter levado o bebê para a primeira consulta de rotina de seguimento e 21,8\% (IC95\%: 20,2-23,5) relataram que esta consulta foi realizada na primeira semana de vida. De forma semelhante ao observado em relação à procura da consulta de pós-parto, valores significativamente mais altos de procura de serviço para consulta do recém-nascido foram observados em mulheres residentes nas regiões Sul, Sudeste e Centro-oeste, com maior escolaridade, com plano de saúde, com assistência pré-natal adequada e que receberam orientação na maternidade para procurar um serviço de pós-parto. Maior procura de consulta para o recém-nato também foi observada em mulheres pertencentes às classes econômicas mais altas e em primíparas, e menor procura em adolescentes (Tabela 3). Para a obtenção da consulta na primeira semana de vida do recém-nascido, além dos fatores anteriores associados à maior procura, cabe destacar o baixo valor do indicador em mulheres que se declararam indígenas, de apenas 2,7\%, valor muito inferior ao referido pelas demais mulheres (Tabela 3).

Para os indicadores relacionados à vacinação, verificou-se uma cobertura vacinal de 99\% (IC95\%: 98,7-99,2) para a vacina BCG e de 96,8\% (IC95\%: 96,0-97,5) para a primeira dose da vacina de hepatite B. Coberturas significativamente menores da vacina BCG foram observadas em primíparas, e significativamente maiores em mulheres com plano de saúde e com Ensino Fundamental completo (Tabela 4). Para a vacina da hepatite B, as menores coberturas foram observadas em mulheres residen- 
Tabela 5

Fatores associados à realização e recebimento do resultado do teste de triagem neonatal. Brasil, 2011-2012.

\begin{tabular}{|c|c|c|c|c|c|c|c|c|c|c|}
\hline \multirow[t]{2}{*}{ Característica da mulher } & \multicolumn{5}{|c|}{$\begin{array}{l}\text { Realização do teste do pezinho nos primeiros } \\
\qquad \begin{array}{l}7 \text { dias de vida } \\
(\mathrm{N}=\mathbf{2 3 . 3 6 8})\end{array}\end{array}$} & \multicolumn{5}{|c|}{$\begin{array}{l}\text { Recebimento do resultado do teste do pezinho } \\
\text { no } 1 \text { o mês de vida } \\
(\mathrm{N}=23.368)\end{array}$} \\
\hline & $\%$ & $\mathrm{RCb}$ & IC95\% & $\mathrm{RCa}$ & IC95\% & $\%$ & $\mathrm{RCb}$ & IC95\% & $\mathrm{RCa}$ & IC95\% \\
\hline Total & 60,1 & & & & & 29,8 & & & & \\
\hline \multicolumn{11}{|l|}{ Região } \\
\hline Norte & 38,2 & 1,00 & * & 1,00 & * & 23,1 & 1,00 & * & 1,00 & ** \\
\hline Nordeste & 36,0 & 0,91 & $0,67-1,24$ & 0,89 & $0,65-1,22$ & 24,7 & 1,10 & $0,77-1,56$ & 0,94 & $0,66-1,34$ \\
\hline Sudeste & 77,1 & 5,45 & $4,00-7,42$ & 5,21 & $3,82-7,11$ & 31,5 & 1,53 & $1,10-2,14$ & 1,08 & $0,78-1,51$ \\
\hline Sul & 72,1 & 4,17 & $2,81-6,19$ & 3,93 & $2,64-5,85$ & 36,6 & 1,93 & $1,34-2,77$ & 1,21 & $0,84-1,77$ \\
\hline Centro-oeste & 63,9 & 2,86 & $1,87-4,36$ & 2,65 & $1,72-4,09$ & 37,7 & 2,01 & $1,43-2,83$ & 1,53 & $1,09-2,14$ \\
\hline \multicolumn{11}{|l|}{ Idade (anos) } \\
\hline 35 ou mais & 61,1 & 1,00 & ** & & & 36,5 & 1,00 & * & & \\
\hline $20-34$ & 61,2 & 1,01 & $0,85-1,19$ & & & 30,4 & 0,76 & $0,65-0,88$ & & \\
\hline $12-19$ & 55,5 & 0,79 & $0,66-0,96$ & & & 24,0 & 0,55 & $0,45-0,67$ & & \\
\hline \multicolumn{11}{|l|}{ Cor da pele autorreferida } \\
\hline Branca & 65,5 & 1,00 & * & & & 37,1 & 1,00 & * & 1,00 & ** \\
\hline Parda & 57,4 & 0,71 & $0,62-0,81$ & & & 26,7 & 0,62 & $0,54-0,71$ & 0,86 & $0,76-0,98$ \\
\hline Preta & 57,0 & 0,70 & $0,56-0,87$ & & & 23,1 & 0,51 & $0,54-0,65$ & 0,79 & $0,63-0,99$ \\
\hline Amarela & 61,4 & 0,84 & $0,55-1,26$ & & & 23,6 & 0,53 & $0,34-0,81$ & 0,60 & $0,39-0,91$ \\
\hline Indígena & 52,6 & 0,58 & $0,33-1,04$ & & & 21,1 & 0,45 & $0,24-0,88$ & 0,70 & $0,33-1,46$ \\
\hline \multicolumn{11}{|l|}{ Escolaridade } \\
\hline $\begin{array}{l}\text { Ensino Fundamental } \\
\text { incompleto }\end{array}$ & 52,0 & 1,00 & * & 1,00 & * & 21,2 & 1,00 & $* *$ & 1,00 & * \\
\hline $\begin{array}{l}\text { Ensino Fundamental } \\
\text { completo }\end{array}$ & 60,2 & 1,40 & $1,18-1,66$ & 1,23 & $1,04-1,44$ & 25,0 & 1,24 & $1,05-1,45$ & 1,07 & $0.90-1,27$ \\
\hline Ensino Médio completo & 63,5 & 1,61 & $1,37-1,88$ & 1,35 & $1,16-1,57$ & 31,9 & 1,74 & $1,46-2,07$ & 1,16 & $0,97-1,39$ \\
\hline $\begin{array}{l}\text { Ensino Superior completo } \\
\text { e mais }\end{array}$ & 65,7 & 1,77 & $1,43-2,21$ & 1,50 & $1,18-1,90$ & 56,4 & 4,81 & $3,82-6,06$ & 1,94 & $1,54-2,45$ \\
\hline \multicolumn{11}{|l|}{ Primípara } \\
\hline Não & 60,0 & 1,00 & $\star \star *$ & & & 27,3 & 1,00 & * & 1,00 & $* *$ \\
\hline Sim & 60,2 & 1,01 & $0,91-1,11$ & & & 32,5 & 1,29 & $1,18-1,41$ & 1,18 & $1,07-1,30$ \\
\hline \multicolumn{11}{|l|}{ Classe econômica } \\
\hline $\mathrm{D} / \mathrm{E}$ & 49,1 & 1,00 & * & & & 21,1 & 1,00 & * & 1,00 & * \\
\hline C & 61,2 & 1,64 & $1,43-1,88$ & & & 26,3 & 1,33 & $1,14-1,55$ & 1,01 & $0,86-1,18$ \\
\hline $\mathrm{A} / \mathrm{B}$ & 67,5 & 2,15 & $1,78-2,61$ & & & 45,0 & 3,06 & $2,48-3,77$ & 1,32 & $1,09-1,60$ \\
\hline \multicolumn{11}{|l|}{ Plano de saúde } \\
\hline Não & 57,9 & 1,00 & * & & & 22,8 & 1,00 & * & 1,00 & * \\
\hline Sim & 65,9 & 1,40 & $1,20-1,64$ & & & 48,7 & 3,23 & $2,73-3,82$ & 2,20 & $1,88-2,56$ \\
\hline \multicolumn{11}{|l|}{ Adequação do pré-natal } \\
\hline Inadequado & 54,7 & 1,00 & * & 1,00 & ** & 25,0 & 1,00 & * & & \\
\hline Adequado & 62,9 & 1,41 & $1,28-1,55$ & 1,16 & $1,05-1,29$ & 32,0 & 1,41 & $1,23-1,61$ & & \\
\hline \multicolumn{11}{|l|}{$\begin{array}{l}\text { Orientada a comparecer a } \\
\text { serviço de pós-parto }\end{array}$} \\
\hline Não & 52,1 & 1,00 & * & & & 22,7 & 1,00 & * & 1,00 & * \\
\hline Sim & 63,5 & 1,61 & $1,41-1,83$ & & & 33,0 & 1,68 & $1,45-1,93$ & 1,38 & $1,21-1,57$ \\
\hline
\end{tabular}

IC95\%: intervalo de 95\% de confiança; RCa: razão de chances ajustada; RCb: razão de chances bruta.

* $p<0,001$;

$* * p<0,05$;

$* * * p>0,20$. 
tes na Região Sudeste, e as maiores naquelas com Ensino Fundamental completo ou Ensino Médio. Cabe ressaltar que, apesar das diferenças significativas encontradas para algumas variáveis, todas as coberturas vacinais foram superiores a $95 \%$ (Tabela 4).

Quanto à realização do teste do pezinho, 60,1\% (IC95\%: 57,6-62,6) das mulheres referiram que a coleta foi realizada na primeira semana de vida e 29,8\% (IC95\%: 27,6-32,2) informaram que receberam o resultado do teste no primeiro mês de vida da criança. A realização do exame no período adequado esteve associada à residência nas regiões Sul, Sudeste e Centro-oeste, à maior escolaridade e a ter tido uma assistência pré-natal adequada (Tabela 5). Já o recebimento do resultado no primeiro mês de vida esteve associado a ser residente na Região Centro-oeste, ter Ensino Superior, ser primípara, pertencer à classe econômica A/B, possuir plano de saúde e ter recebido orientação para comparecer ao serviço de saúde. Menor recebimento do resultado do exame foi observado em mulheres de cor não branca (Tabela 5).

\section{Discussão}

Dos oito indicadores de saúde relacionados à utilização de serviços de saúde após o parto, apenas quatro ("procura de serviço para consulta de revisão do parto", "procura de serviço de saúde para realização da consulta de rotina de acompanhamento do recém-nascido", "vacinação do recém-nascido com a vacina BCG" e "vacinação do recém-nascido com a primeira dose da vacina contra hepatite B”) apresentaram valores superiores a $75 \%$ na estimativa intervalar, sendo considerados satisfatórios. Um indicador ("realização do teste do pezinho nos primeiros sete dias de vida do recém-nascido") apresentou valor entre 50\% e 75\%, com implantação parcial, e três indicadores ("realização de consulta de revisão do parto nos primeiros 15 dias pós-parto", "realização da primeira consulta de rotina de seguimento do recém-nascido na primeira semana de vida" e "recebimento dos resultados do teste do pezinho no primeiro mês de vida do recém-nascido") foram considerados insatisfatórios, por terem apresentado valor inferior a $50 \%$.

A cobertura satisfatória de indicadores de vacinação pode ser decorrente da consolidação do Programa Nacional de Imunização, implantado em 1973, que historicamente apresenta coberturas elevadas no país (Departamento de Informática do SUS. Arquivos de DO reduzida para tabulação do Programa Nacional de Imunização. http://tabnet.datasus.gov.br/cgi/deftohtm.exe?pni/cnv/cpniuf.def, acessado em 05/Dez/2018). Adicionalmente, o programa de transferência condicional de renda Bolsa Família pode ter contribuído para esse resultado, de forma similar ao efeito positivo nas coberturas vacinais em beneficiários de programas de transferência de renda encontrado em outros países 26. O programa Bolsa Família apresenta, entre as condicionalidades para a permanência da família no programa, a manutenção do calendário de vacinação das crianças menores de sete anos em dia, além de visitas frequentes ao posto de saúde para que sejam pesadas, medidas e tenham o crescimento monitorado 27 . Um estudo recente encontrou maior cobertura vacinal quando a criança e/ou sua mãe recebem outras intervenções de saúde, demostrando a importância de oportunizar os contatos com os serviços para a realização da vacinação 28 .

Embora a procura para a consulta de revisão pós-parto tenha atingido nível satisfatório, seu valor foi muito inferior ao observado para a busca da consulta de rotina de acompanhamento do recém-nascido, indicando que em muitas situações a mulher comparece ao serviço de saúde para atendimento ao bebê, mas não para atenção à sua própria saúde. Um dos principais determinantes da utilização de serviços é a necessidade percebida de cuidado 29 , e uma possível explicação para a menor procura da consulta de revisão do parto seria o desconhecimento das mulheres sobre a importância dessa consulta de revisão. A menor valorização da consulta de puerpério pode ser reforçada pela própria prática dos serviços. Um estudo qualitativo realizado em Recife, Pernambuco, evidenciou insatisfação das mulheres com o atendimento pós-parto, por desvalorização das necessidades de saúde femininas, com atenção focada no bebê, escassez de exame físico e anamnese, orientações insuficientes e comunicação limitada 30 . Neste estudo, o fator mais fortemente associado à procura e obtenção da consulta de revisão após o parto foi o recebimento, na maternidade, de orientação para o comparecimento ao serviço de pós-parto. De forma similar, a falta de informação sobre serviços pós-natais foi o principal motivo de não utilização desses serviços num estudo realizado na Etiópia ${ }^{31}$. A orientação recebida 
na maternidade também foi associada à maior procura e recebimento da consulta de recém-nato e ao recebimento do exame de triagem neonatal no primeiro mês de vida.

Os indicadores com coberturas mais baixas e considerados insatisfatórios foram aqueles que dependem da estrutura de funcionamento dos serviços de atenção básica, incluindo consultas e realização de exames. Ressalte-se que a coleta do teste do pezinho apresentou implantação parcial, o que pode ter sido decorrente de sua realização também em nível hospitalar, antes da alta após a internação para o parto.

A comparação dos dados da consulta de puerpério com os resultados do único estudo disponível no âmbito nacional, a Pesquisa Nacional de Demografia em Saúde (PNDS), realizada em 2006 , é limitada pelo indicador utilizado: realização de ao menos uma consulta de puerpério até os 42 dias no parto na PNDS, e nos primeiros 15 dias após o parto neste estudo. Considerando-se essa limitação, podemos verificar que o acesso global não se modificou substancialmente (39,2\% em 2006, 37\% em 2011/2012), mas houve aumento na realização de consulta de puerpério por mulheres residentes na Região Norte, passando de 19,2\% para 25,6\%, com redução da desigualdade regional. A redução da desigualdade social no acesso a serviços de atenção à saúde da mulher e da criança no Brasil tem sido relatada por outros autores $27,32,33$, bem como o papel da atenção primária em saúde na maior efetividade dos serviços 11 e na redução da mortalidade infantil 34 .

Dados nacionais relativos à primeira consulta do recém-nato não estão disponíveis para comparação, mas o resultado encontrado de apenas um quinto dos recém-nascidos com consulta realizada na primeira semana de vida é preocupante. A procura para a realização da consulta do recém-nato foi elevada, superior a 90\%, demonstrando que existe uma necessidade percebida de cuidado, e que, provavelmente, barreiras de acesso estão impedindo a utilização desse serviço no prazo recomendado. Uma hipótese alternativa é a de que a procura do serviço não tenha sido precoce, já que a pergunta feita às mulheres não delimitava a época em que o serviço foi procurado. Um estudo anterior realizado na Região Sul do Brasil identificou que grande parte do atendimento a menores de um ano é demandado por motivo de doença da criança e não para o acompanhamento do processo de crescimento e desenvolvimento infantil 10 .

Em relação à triagem neonatal, indicadores do Programa Nacional de Triagem Neonatal (PNTN) 9 demonstram cobertura de realização do teste do pezinho igual ou superior a 80\% desde o ano de 2005, porém com coleta na data adequada entre 45\% e 62\% no período 2004-2017. No ano de 2011, ano de realização da maior parte das entrevistas deste estudo, o valor reportado pelo PNTN foi de 61,97\% 9 , semelhante ao encontrado nesta pesquisa. Ressalta-se que, a partir de 2011, o PNTN passou a recomendar a coleta da amostra até o 5o dia de vida, devido à inclusão de novas doenças no programa.

Dados sobre a data de emissão dos resultados não estão disponíveis dentre os indicadores monitorados pelo PNTN. O indicador "mediana da idade do recém-nascido na data da primeira consulta", avaliado para cada doença, variou de 30 a 50 dias no ano de $2011^{9}$, indicando que metade das crianças iniciou acompanhamento médico após este período, o que pode ser determinado pela demora no recebimento do resultado e/ou no acesso aos médicos especialistas. O resultado encontrado neste estudo, de que apenas um terço dos recém-natos recebeu o resultado do teste do pezinho no primeiro mês de vida, indica ser esta uma barreira importante para o tratamento oportuno da doença identificada e a prevenção de complicações e sequelas.

A PNS realizada em 2013 avaliou a triagem neonatal em menores de dois anos com base no relato da mãe, sendo estimada cobertura de $96,5 \%$ de triagem neonatal considerando qualquer idade da criança 35 . Essa diferença no critério adotado pode explicar o valor mais elevado em relação ao nosso estudo, que considerou apenas a coleta e o recebimento no prazo recomendado. Na PNS, foi observada maior cobertura da triagem neonatal nas regiões Sul e Sudeste, em usuários de plano de saúde e em famílias com maior renda, com efeito dose resposta 35. Desigualdades regionais e socioeconômicas também foram observadas neste estudo, tanto para a coleta quanto para o recebimento do resultado do exame, com mulheres que possuíam plano de saúde apresentando o dobro de recebimento do resultado do exame no prazo recomendado.

A baixa utilização e as disparidades identificadas no uso dos serviços de saúde por puérperas e recém-nascidos são condizentes com achados de estudos nacionais 5,6,7,10,11,15 e internacionais anteriores 14,16,17,18,20,21,36,37. Desigualdades regionais foram observadas para todos os indicadores, bem como a associação da maior escolaridade materna ao maior uso dos serviços, independentemente da 
classe econômica e da disponibilidade de plano de saúde. Cabe ressaltar a baixa cobertura de consultas entre as indígenas, tanto para a consulta da mulher quanto do recém-nascido, revelando barreiras específicas enfrentadas por este grupo populacional. Baixa cobertura de consultas e exames de prénatal também foi identificada no Primeiro Inquérito Nacional de Saúde e Nutrição dos Povos Indígenas 38. Ressalta-se também o efeito independente da assistência pré-natal adequada, principalmente para os cuidados maternos, mas também para a procura da consulta do recém-nascido e para a coleta do exame de triagem neonatal na época recomendada. O efeito do cuidado pré-natal na maior utilização de serviços pós-natais já foi demonstrado tanto em mulheres adultas 14,17,20,21,36 quanto em adolescentes 18. O efeito positivo da assistência pré-natal e das orientações recebidas na maternidade para os cuidados pós-natais demostra a importância da integração dos serviços de saúde, para a garantia da continuidade do cuidado durante o ciclo gravídico-puerperal, contribuindo para a redução das inequidades no acesso aos serviços e para a maior efetividade da assistência à saúde das mulheres e recém-natos.

Este estudo apresenta algumas limitações. Foram incluídas apenas puérperas atendidas em hospitais com mais de 500 partos/ano, o que corresponde a $80 \%$ dos nascimentos ocorridos no país. Entretanto, é possível que mulheres com partos em hospitais menores, ou com partos domiciliares ou em via pública tenham um perfil diferente de utilização dos serviços de saúde. Também não foram incluídas mulheres com gestações gemelares, com óbito fetal ou neonatal como desfecho da gestação, que permaneceram internadas por mais de 15 dias, bem como recém-natos que ficaram internados por mais de sete dias. Ainda que essas situações sejam pouco frequentes, e provavelmente não afetem as estimativas apresentadas, apontam para a necessidade de estudos futuros que avaliem a utilização de serviços por esses grupos específicos.

Todas as informações usadas na análise foram referidas pelas mulheres, sem confirmação em registros hospitalares ou nos cartões da gestante e da criança, estando sujeitas a viés de informação. Como o período de realização da entrevista foi amplo, com $25 \%$ das entrevistas realizadas mais de 4 meses após o parto, existe a possibilidade de viés de memória, principalmente para as mulheres entrevistadas mais tardiamente, o que pode ter afetado a qualidade da resposta.

Alguns procedimentos, como vacinação e coleta do teste do pezinho, também podem ser feitos na maternidade antes da alta hospitalar, e nossos resultados podem ter superestimado o desempenho dos serviços ambulatoriais, embora não saibamos com que magnitude.

Foram calculados apenas os indicadores de utilização dos serviços, sem avaliação da qualidade do atendimento. É importante que estudos futuros avaliem também a adequação do conteúdo das consultas, visando a verificar se mulheres e recém-nascidos estão tendo acesso às melhores práticas assistenciais recomendadas pelos protocolos assistenciais disponíveis.

Para o indicador relativo à realização de consulta de puerpério, a norma técnica atual, recomendada pelo Ministério da Saúde 2, é de que a mulher tenha contato com o serviço de saúde entre 7 e 10 dias após o parto, podendo a consulta puerperal ser agendada até 42 dias após o parto. Em nossa pesquisa, a informação disponível refere-se à realização da consulta de puerpério nos primeiros 15 dias após o parto. É possível que a utilização do parâmetro recomendado pelo Ministério da Saúde resultasse em proporções mais elevadas de comparecimento à consulta. Além disso, não dispomos de informações sobre visitas domiciliares, realização de triagem auditiva nem de ações voltadas para a orientação e o apoio ao aleitamento materno. Esses itens fazem parte do conjunto de ações recomendadas para serem realizadas na "Primeira Semana de Saúde Integral". Estudos futuros deverão incluir esses itens para uma avaliação completa das ações recomendadas para a atenção à saúde do binômio mãe/bebê na primeira semana após o parto. 


\section{Conclusão}

Apenas quatro indicadores de utilização de serviços após o parto apresentaram avaliação satisfatória, persistindo desigualdades regionais e sociais, com populações mais vulneráveis apresentando os piores indicadores. Proporções mais elevadas e com menos desigualdade foram observadas para os indicadores relacionados à vacinação dos recém-natos, provável reflexo do Programa Nacional de Imunização implantado há mais de 40 anos no país. Indicadores que incluem prazo de realização e que dependem exclusivamente da atenção básica apresentaram o pior desempenho, indicando a necessidade de uma melhor organização e oferta dos serviços visando à redução de barreiras de acesso. Estudos futuros deverão investigar os tipos de barreira relatados pelas mulheres, bem como a qualidade dos serviços que estão sendo utilizados.

\section{Colaboradores}

R. M. S. M. Domingues contribuiu na concepção, análise dos dados e redação da versão final a ser publicada. B. A. S. Dias contribuiu na concepção, análise dos dados, revisão e aprovação da versão final a ser publicada. S. D. A. Bittencourt e M. A. B. Dias contribuíram na concepção, redação da versão inicial e aprovação final da versão a ser publicada. J. A. Torres, E. M. Cunha e M. C. Leal contribuíram na revisão e aprovação final da versão a ser publicada.

\section{Informações adicionais}

ORCID: Rosa Maria Soares Madeira Domingues (0000-0001-5722-8127); Barbara Almeida Soares Dias (0000-0001-8656-1391); Sonia Duarte de Azevedo Bittencourt (0000-0003-2466-1797); Marcos Augusto Bastos Dias (0000-0003-1386-7001); Jacqueline Alves Torres (0000-0002-0567-2952); Elenice Machado da Cunha (0000-0001-7471-301X); Maria do Carmo Leal (0000-0002-3047-515X).

\section{Agradecimentos}

Ao Conselho Nacional de Desenvolvimento Científico e Tecnológico; Ministério da Saúde; Escola Nacional de Saúde Pública Sergio Arouca, Fundação Oswaldo Cruz; Fundação de Amparo à Pesquisa do Estado do Rio de Janeiro.

\section{Referências}

1. American College of Obstetricians and Gynecologists. Postpartum toolkit. 2018. https:// www.acog.org/-/media/Departments/Tool kits-for-Health-Care-Providers/Postpartum-/ Dezkit/2018-Postpartum-Toolkit.pdf? dmc $=1$ $\&$ ts $=20190206 \mathrm{~T} 1216441106$ (acessado em 05/ Dez/2018).

2. Departamento de Atenção Básica, Secretaria de Atenção à Saúde, Ministério da Saúde. Atenção ao pré-natal de baixo risco. Brasília: Editora do Ministério da Saúde; 2012. (Série A. Normas e Manuais Técnicos) (Cadernos de Atenção Básica, 32).

3. Departamento de Ações Programáticas Estratégicas, Secretaria de Atenção à Saúde, Ministério da Saúde. Agenda de compromissos para a saúde integral da criança e redução da mortalidade infantil. http://bvsms.saude.gov.br/ bvs/publicacoes/agenda_compro_crianca.pdf (acessado em 30/Out/2019).

4. Viellas EF, Domingues RMSM, Dias MAB, Gama SGN, Theme-Filha MM, Costa JV et al. Assistência pré-natal no Brasil. Cad Saúde Pública 2014; 30 Suppl 1:S85-100.

5. Ministério da Saúde. Pesquisa Nacional de Demografia e Saúde da Criança e da Mulher - PNDS 2006: dimensões do processo reprodutivo e da saúde da criança. http://bvsms.sau de.gov.br/bvs/publicacoes/pnds_crianca_mu lher.pdf (acessado em 12/Jun/2019).

6. Hass CNC, Teixeira LB, Beghetto MG. Adequacy of prenatal care in a family health strategy program from Porto Alegre - RS. Rev Gaúcha Enferm 2013; 34:22-30. 
7. Matijasevich A, Santos IS, Silveira MF, Domingues MR, Barros AJ, Marco PL, et al. Inequities in maternal post-natal visits among public and private patients: 2004 Pelotas cohort study. BMC Public Health 2009; 9:335.

8. Oliveira RLA, Fonseca CRB, Carvalhaes MABL, Parada CMGL. Avaliação da atenção pré-natal na perspectiva dos diferentes modelos na atenção primária. Rev Latinoam Enferm 2013; 21:546-53.

9. Ministério da Saúde. Programa Nacional de Triagem Neonatal. http://portalms.saude. gov.br/acoes-e-programas/programa-nacio nal-da-triagem-neonatal/indicadores-datriagem-neonatal-no-brasil (acessado em 14/ Fev/2019).

10. Vitolo MR, Gama, CM, Campagnolo PDB. Frequência de utilização do serviço público de puericultura e fatores associados. J Pediatr (Rio J.) 2010; 86:80-4.

11. Piccini RX, Facchini LA, Tomasi E, Thumé E, Silveira DS, Siqueira FV, et al. Efetividade da atenção pré-natal e de puericultura em unidades básicas de saúde do Sul e do Nordeste do Brasil. Rev Bras Saúde Matern Infant 2007; 7:75-82.

12. Travassos C, Martins M. Uma revisão sobre os conceitos de acesso e utilização de serviços de saúde. Cad Saúde Pública 2004; 20 Suppl 2:S190-8.

13. Stopa SR, Malta DC, Monteiro CN, Szwarcwald CL, Goldbaum M, Cesar CLG. Use of and access to health services in Brazil, $2013 \mathrm{Na}$ tional Health Survey. Rev Saúde Pública 2017; 51 Suppl 1:3s.

14. Tarekegn SM, Lieberman LS, Giedraitis V. Determinants of maternal health service utilization in Ethiopia: analysis of the 2011 Ethiopian Demographic and Health Survey. BMC Pregnancy Childbirth 2014; 14:161.

15. Gonçalves CS, Cesar JA, Marmitt LP, Gonçalves $\mathrm{CV}$. Frequência e fatores associados a não realização da consulta puerperal em um estudo de coorte. Rev Bras Saúde Mater Infant 2019; 19:71-8

16. Gu H, You H, Yan Z, Yang N, Kou Y, Sun J, et al. Determinants of the utilization of postpartum family visits: Evidence from rural areas of Eastern China. PLoS One 2018; 13:e0194061.

17. Khanal V, Adhikari M, Karkee R, Gavidia T. Factors associated with the utilisation of postnatal care services among the mothers of $\mathrm{Ne}$ pal: analysis of Nepal demographic and health survey 2011. BMC Womens Health 2014; 14:19.

18. Banke-Thomas OE, Banke-Thomas AO, Ameh CA. Factors influencing utilisation of maternal health services by adolescent mothers in Lowand middle-income countries: a systematic review. BMC Pregnancy Childbirth 2017; 17:65.

19. Bryant A, Blake-Lamb T, Hatoum I, Kotelchuck M. Women's use of health care in the first 2 years postpartum: occurrence and correlates. Matern Child Health J 2016; 20 Suppl $1: 81-91$.
20. Fekadu GA, Kassa GM, Berhe AK, Muche AA, Katiso NA. The effect of antenatal care on use of institutional delivery service and postnatal care in Ethiopia: a systematic review and meta-analysis. BMC Health Serv Res 2018; 18:577.

21. Fekadu GA, Ambaw F, Kidanie SA. Facility delivery and postnatal care services use among mothers who attended four or more antenatal care visits in Ethiopia: further analysis of the 2016 demographic and health survey. BMC Pregnancy Childbirth 2019; 19:64.

22. Vasconcelos MTL, Silva PLN, Pereira APE, Schilithz AOC, Souza Junior PRB, Szwarcwald CL. Desenho da amostra Nascer no Brasil: Pesquisa Nacional sobre Parto e Nascimento. Cad Saúde Pública 2014; 30 Suppl 1:S49-58.

23. Leal MC, Silva AAM, Dias MAB, Gama SGN, Rattner D, Moreira ME, et al. Birth in Brazil: national survey into labour and birth. Reprod Health 2012; 9:15.

24. Alves CKA, Natal S, Felisberto E, Samico I. Interpretação e análise das informações: o uso de matrizes, critérios, indicadores e padrões. In: Samico I, Felisberto E, Figueiró AC, Frias PG, organizadores. Avaliação em saúde: bases conceituais e operacionais. Rio de Janeiro: MedBook; 2010. p. 89-107.

25. Associação Brasileira de Empresas de Pesquisa. Critério padrão de classificação econômica Brasil/2008. http://www.aba.com.br/wp-con tent/uploads/content/7727632a373615b $34 \mathrm{f} 2 \mathrm{a} 5726 \mathrm{fcc} 5 \mathrm{c} 9 \mathrm{e} 2$.pdf (acessado em 10/Out/ 2019).

26. Cruz RCS, Moura LBA, Soares Neto JJ. Conditional cash transfers and the creation of equal opportunities of health for children in low and middle-income countries: a literature review. Int J Equity Health 2017; 16:161.

27. Leal MDC, Szwarcwald CL, Almeida PVB, Aquino EML, Barreto ML, Barros F, et al. Reproductive, maternal, neonatal and child health in the 30 years since the creation of the Unified Health System (SUS). Ciênc Saúde Colet 2018; 23:1915-28.

28. Restrepo-Méndez MC, Barros AJ, Wong KL, Johnson HL, Pariyo G, Wehrmeister FC, et al. Missed opportunities in full immunization coverage: findings from low- and lowermiddle-income countries. Glob Health Action 2016; 9:30963.

29. Andersen RM. Revisiting the behavioral model and access to medical care: does it matter? J Soc Behav 1995; 36:1-10.

30. Corrêa MSM, Feliciano KVO, Pedrosa EN, Souza AI. Acolhimento no cuidado à saúde da mulher no puerpério. Cad Saúde Pública 2017; 33:e00136215.

31. Abota TL, Atenafu NT. Postnatal care utilization and associated factors among married women in Benchi-Maji Zone, Southwest Ethiopia: a community based cross-sectional study. Ethiop J Health Sci 2018; 28:267-6. 
32. Cesar JA, Matijasevich A, Santos IS, Barros AJ, Dias-da-Costa JS, Barros FC, et al. The use of maternal and child health services in three population-based cohorts in Southern Brazil, 1982-2004. Cad Saúde Pública 2008; 24 Suppl 3:S427-36.

33. Barros FC, Matijasevich A, Requejo JH, Giugliani E, Maranhão AG, Monteiro CA, et al. Recent trends in maternal, newborn, and child health in Brazil: progress toward Millennium Development Goals 4 and 5. Am J Public Health 2010; 100:1877-89.

34. Russo LX, Scott A, Sivey P, Dias J. Primary care physicians and infant mortality: evidence from Brazil. PLoS One 2019; 14:e217614.

35. Mallmann MB, Tomasi YT, Boing AF. Neonatal screening tests in Brazil: prevalence rates and regional and socioeconomic inequalities. J Pediatr (Rio J.) 2019; [Epub ahead of print].
36. Kanté AM, Chung CE, Larsen AM, Exavery A, Tani K, Phillips JF. Factors associated with compliance with the recommended frequency of postnatal care services in three rural districts of Tanzania. BMC Pregnancy Childbirth $2015 ; 15: 341$

37. Langlois ÉV, Miszkurka M, Zunzunegui MV, Ghaffar A, Ziegler D, Karp I. Inequities in postnatal care in low- and middle-income countries: a systematic review and meta-analysis. Bull World Health Organ 2015; 93:259$70 \mathrm{G}$.

38. Garnelo L, Horta BL, Escobar AL, Santos RV, Cardoso AM, Welch JR, et al. Avaliação da atenção pré-natal ofertada às mulheres indígenas no Brasil: achados do Primeiro Inquérito Nacional de Saúde e Nutrição dos Povos Indígenas. Cad Saúde Pública 2019; 35 Suppl 3:e00181318. 


\section{Abstract}

This study aims to estimate postpartum use of outpatient health services and verify the demographic, socioeconomic, and obstetric factors associated with use. A nationwide hospital-based study in 2011-2012 interviewed 23,894 women. Point estimates and respective confidence intervals were calculated for eight indicators of health services use, with performance assessed as "satisfactory" (75\%-100\%); "partial” (50\%-74\%), or "unsatisfactory" (<50\%). Multiple logistic regression was performed to verify the association between women's characteristics and each target indicator. Four indicators ("visit to health service for postpartum follow-up" (73.9\%; 95\%CI: 72.4-75.3), "visit to health service for neonatal follow-up" (91.6\%; 95\%CI: 90.6-92.5), "BCG vaccination" (99\%; 95\%CI: 98.7-99.2), and "HBV vaccination" (96.8\%; 95\%CI: 96.0-97.5) were considered satisfactory. "Neonatal screening test in the first week of life" was considered partial (60.1\%; 95\%CI: 57.6-62.6), while "woman's consultation in the first 15 days postpartum" (37\%; 95\% CI: 35.0-39.0), "neonatal consultation in the first seven days of life" (21.8\%; 95\%CI: 20.2-23.5), and "neonatal screening test result in the first month of life" (29.8\%; 95\%CI: 27.6-32.2) were considered unsatisfactory. Regional and social inequalities were identified, with worse performance for all the indicators in the North and Northeast regions of Brazil and in more vulnerable women, revealing the need for better organization and supply of services to reduce iniquities.

Maternal and Child Health; Maternal-Child Health Services; Child Care; Postpartum Period

\section{Resumen}

El objetivo de este estudio es estimar la utilización de los servicios de salud ambulatorios durante el posparto, así como verificar los factores demográficos, socioeconómicos y obstétricos, asociados a esta utilización. Estudio nacional de base hospitalaria, realizado en 2011-2012, con una entrevista a 23.894 mujeres. Se calcularon las estimaciones puntuales y los respectivos intervalos de confianza de ocho indicadores de utilización de servicios de salud con desempeño evaluado como "satisfactorio" (75\%-100\%); "parcial" (50\%-74\%) e "insatisfactorio" (< 50\%). Se realizó una regresión logística múltiple para verificar la asociación entre las características de las mujeres y cada uno de los indicadores analizados. Cuatro indicadores - "búsqueda del servicio para consulta de revisión del parto" (73,9\%; IC95\%: 72,4-75,3), "búsqueda del servicio para consulta del recién nacido" (91,6\%; IC95\%: 90,6-92,5), "vacunación con BCG" (99\%; IC95\%: 98,7-99,2) y "vacunación contra hepatitis B" (96,8\%; IC95\%: 96,0-97,5) se consideraron satisfactorios. La "recogida del test de clasificación neonatal en la primera semana de vida" se consideró parcial (60,1\%; IC95\%: 57,6-62,6), mientras que "consulta de la mujer durante los primeros 15 dias tras el parto" (37\%; IC95\%: 35,0-39,0), "consulta del recién nacido en los primeros siete días de vida" (21,8\%; IC95\%: 20,2-23,5) y "recepción del resultado de la clasificación neonatal durante el primer mes de vida" (29,8\%; IC95\%: 27,6-32,2) fueron considerados insatisfactorios. Las desigualdades regionales y sociales se identificaron con un peor desempeño de todos los indicadores en las regiones Norte y Nordeste, y en mujeres más vulnerables, apuntando la necesidad de una mejor organización y oferta de los servicios, con el fin de reducir de inequidades.

Salud Materno-Infantil; Servicios de Salud Materno-Infantil; Cuidado del Niño;

Periodo Posparto
Recebido em 25/Jun/2019

Versão final reapresentada em 31/Out/2019

Aprovado em 06/Nov/2019 